CM-P00062459

CERN-TH.5993/91

$\mathrm{SPhT} / 91-008$

\title{
SPIKES, ANOMALOUS EVENTS AND THE DIMENSIONAL STRUCTURE OF MULTIPARTICLE FLUCTUATIONS
}

\author{
Ph. Brax, R. Peschanski \\ Service de Physique Théorique ${ }^{\dagger}$ \\ de Saclay \\ 91191 Gif-sur-Yvette Cedex, France \\ and \\ CERN, Theoretical Physics Division* \\ 1211 Geneva 23, Switzerland
}

\begin{abstract}
Random cascading models are used to describe intermittency patterns of multiparticle events in high energy reactions. The effect of projecting $D$-dimensional cascades onto $(D-1)$ dimensions is tackled. It leads to a non trivial dimensional classificaton of projected events into three classes: bulk intermittent events, spikes corresponding to a $D$-dimensional phase transition and eventually anomalous events related to sheer $(D-1)$ dimensional patterns. Applications to $\mathrm{e}^{+}-\mathrm{e}^{-}$and $h-h$ reactions are envisaged using the Levy-stable law intermittency parametrization.
\end{abstract}

† Laboratoire de la Direction des Sciences de la Matière du Commissariat à l'Energie Atomique.

* Adress until September 1991

CERN-TH.5993/91

$\mathrm{SPhT} / 91-008$

January 1991 


\section{I - MOTIVATIONS}

Intermittency, the possibility of dynamically fluctuating patterns in high energy physics, was first noticed in the 1-dimensional analysis performed on the particle distributions along the rapidity axis $^{[1]}$. Within a certain range, scaling laws are obtained, characterized by a power law behaviour

$$
\frac{\left\langle\rho_{m}^{q}\right\rangle}{\left\langle\rho_{m}\right\rangle^{q}}=\varepsilon^{-\varphi(q)}
$$

where $\rho_{m}$ is the multiplicity density in the phase space interval $[m]$, (namely the $m^{\text {th }}$ rapidity bin) whose typical size is $\varepsilon$. The density moments can be reached experimentally using factorial moments of the actually measured multiplicity distribution. The average is taken over event samples.

Beside this quantitative approach, event per event rapidity distributions such as noticed by collaborations NA22 ${ }^{[2]}$ and JACEE ${ }^{[3]}$ (see Fig.1) can be of a puzzling nature. They can look very different as, for example, the latter which is made of many dips and spikes with no particular feature ("bulk intermittency") whereas the former is dominated by a high spike where many particles are clustered within the same rapidity bin. This type of extremely rare events has been coined as an "anomalous event"[2,4].

Recently, it has been pointed out that intermittent systems may undergo a "non-thermal" phase transition during their evolution ${ }^{[5]}$. This "non thermal" phase transition would be responsible for "anomalous events" and would also explain the different types of particle multi-production events $^{[4,5]}$. Using random cascading models (Fig.2), three different kinds of events were identified ${ }^{[6,7]}$. They are represented on the phase diagram (Fig.3). Phase I corresponds to bulk intermittency, phase II contains "anomalous events, i.e. very rare events, whereas phase III is a spin-glass like phase. Thermodynamic quantities such as the free energy and the multifractal spectrum ${ }^{[6]}$ have been calculated. The "non thermal" phase transition is thus explained in terms of a hierarchical phase III where self averingness and ergodicity are explicitly broken.

This gives a clue about the structure of "anomalous events" but does not take into account dimension effects which turn out to be crucial ${ }^{[8]}$. Indeed, it seems more likely that sheer intermittency is to stem from full 2 or 3 dimensional phenomena as it has been confirmed by rapidity-azimuth particle distributions (in 2-dimensions, the element of phase space in (1) is given by $\varepsilon=\delta y \delta \varphi$ where $y$ is the rapidity and $\varphi$ the azimuth). Indeed, one dimensional density moments may not follow a strict power law for small enough rapidity bins. Therefore, it has to be asked what remains from the assumption that "anomalous events" could give a hint about a "non thermal" phase transition.

For instance suppose that there is a $D$-dimensional "non thermal" phase transition, it is relevant to know what "anomalous events" can tell about this transition. One can also wonder what traces of this transition can be found in ordinary 1-dimensional rapidity distributions. We will confirm the remark ${ }^{[8]}$ that for bulk intermittency, projected intermittency is flattened. As will be clear later the three types of events have drastically different projections. In a way, projection reveals the geometrical structure of multiparticle events as well as their thermodynamic features.

The article is arranged as follows. First projected intermittency is studied. It is shown that below a certain threshold $q_{1}$, projected intermittency flattens and the intermittency indices are zero. Thus allows one to classify different kinds of projected systems. This is done by computing multifractal spectra. A structural interpretation of this classification is given in terms of the geometry of events. "Anomalous events" are then discussed as a possible signal of a "non thermal" 
phase transition. Using Levy laws, the above analysis is applied to different reactions such as heavy ions, $\mu p, h p$ and $\mathrm{e}^{+}-\mathrm{e}^{-}$collisions.

\section{II - CUT INTERMITTENCY, PROJECTED INTERMITTENCY}

Factorial moments are used to withdraw the effect of statistical noise and obtain moments of the physical density $\rho_{m}$. We will prove that they can also be used to measure moments of a projected system. Suppose that intermittency is $D$-dimensional, and that phase space (which can be the rapidity $y$ and azimuth $\varphi$ in 2 dimensions) is cut into $M$ pieces. This corresponds to $M=n^{D}$ bins, $n$ being the number of bins along one direction (for instance along the rapidity axis). In each of the $M$ bins, a number $k_{m}$ of particles is measured. When projected along the $i^{\text {th }}$ direction, the number of particles in the $n^{D-1}$ bins is given by

$$
k_{\pi_{j}}=\sum_{m_{j}=1}^{n} k_{m}
$$

where $m=\left(m_{1}, \ldots, m_{D}\right)$ and $\forall i \in\{1, \ldots, D\}, m_{i} \in\{1, \ldots, n\}$.

Recall that the number of particles $k_{m}$ is a particular realization of the dynamical density

$\rho_{m}{ }^{[1]}$. In particle multiproduction it is usually assumed that the probability distribution $Q\left(k_{m}\right)$ of $k_{m}$ 's is the result of a two step process. First there is a probability $P\left(\rho_{m}\right)$ that the density in the $m^{\text {th }}$ bin is $\rho_{m}$, then an average value $\langle N\rangle$ of particles is randomly distributed amongst the $M$ bins. This second process represents the statistical noise which blurs the dynamical density $\rho_{m}$. If each draw of particles around an average of $\langle N\rangle \rho_{m}$ particles per bin is independent of one another, and the number of particles in each bin is sufficiently large, one can model out the blurring by statistical noise with a Poisson law whose expectation value is $\langle N\rangle \rho_{m}$

$$
Q\left(k_{m}\right)=\int \mathrm{d} \rho_{m} \frac{\left(\langle N\rangle \rho_{m}\right)^{k_{m}}}{k_{m} !} \mathrm{e}^{-\langle N\rangle \rho_{m}} P\left(\rho_{m}\right)
$$

It can be argued that for small number of particles, and especially if the total number of particles is fixed, the Poisson law is replaced by a binomial law ${ }^{[9]}$. Let us now prove that disentanglement of statistical noise by factorial moments is preserved by projection. Introduce the generating function $\psi_{\pi_{j}}(Z)$ of the projected number of particles $k_{\pi_{j}}$

$$
\psi_{\pi_{j}}(Z)=\left\langle Z^{k_{\pi_{j}}}\right\rangle_{Q}
$$

where the average is taken over the probability $Q$. Factorial moments of the projected number of particles are given by

$$
\left\langle k_{\pi_{j}}\left(k_{\pi_{j}}-1\right) \ldots\left(k_{\pi_{j}}-q+1\right)\right\rangle=\frac{\mathrm{d}^{q} \psi_{\pi_{j}}}{\mathrm{~d} z^{q}} \mid z=1
$$

From (4), using the mutual independence of $k_{m}$ 's, one gets

$$
\psi_{\pi_{j}}(Z)=\left\langle e^{\langle N\rangle(-1+Z) \sum_{m_{j}=1}^{n} \rho_{m_{j}}}\right\rangle_{P}
$$

where one integrates over the distribution $P$. 
Therefore, taking the appropriate derivatives

$$
\frac{\left\langle k_{\pi_{j}}\left(k_{\pi_{j}}-1\right) \ldots\left(k_{\pi_{j}}-q+1\right)\right\rangle}{\left\langle k_{\pi_{j}}\right\rangle^{q}}=\frac{\left\langle\left(\sum_{m_{j}=1}^{n} \rho_{m_{j}}\right)^{q}\right\rangle}{\left\langle\sum_{m_{j}=1}^{n} \rho_{m_{j}}\right\rangle^{q}}
$$

where $\left\langle\sum_{m} k_{m}\right\rangle=\langle N\rangle$ and $\left\langle\sum_{m} \rho_{m}\right\rangle=1$ has been utilized. Hence factorial moments of the projected number of particles are equal to the normalized moments of the projected density

$$
\rho_{\pi_{j}}=\sum_{m_{j}=1}^{n} \rho_{m_{j}}
$$

In order to investigate intermittent properties of projected systems, we will focus on the projected densities $\rho_{\pi_{j}}$. Statistical properties of these densities can be obtained from the particle distribution $\left\{k_{m}\right\}$ using factorial moments of projected particle numbers $k_{\pi}$. Random cascading models are a very useful tool in order to describe particle densities $\rho_{m}$ and $\rho_{\pi}$. Let us introduce random cascading models.

We are primarily interested in models defined on the $D$-dimensional Cayley tree (see Fig.2). They are described by multiplicative random processes obtained from a building block $W$ in the following manner. Consider $W$ as a random variable whose density $r(W)$ is such that

$$
\int r(W) \mathrm{d} W=\int r(W) W \mathrm{~d} W=1
$$

A realization of this variable is assigned to each link $\alpha$ of the tree. Then denote by $[m]=$ $\left(m_{1}, m_{2}, \ldots, m_{D}\right)$ the $m^{\text {th }}$ end-point of the tree. To each end point $[m]$ corresponds a path $(m)$ emanating from the root of the tree. At each link are assigned $\lambda^{D}$ branches. Thus we define the random density $\rho_{m}$ by

$$
\rho_{m}=\prod_{\alpha \in(m)} \frac{W_{\alpha}}{\lambda^{D}}
$$

where $\left\{W_{\alpha}, \alpha \in(m)\right\}$ is a set of independent realizations of $W$. The intermittency indices are obtained $^{[1]}$ from (10)

$$
\varphi_{D}=\frac{\ln \left\langle W^{q}\right\rangle}{\ln \lambda^{D}}
$$

where the independence of the $W_{\alpha}$ 's and the normalization condition $\langle W\rangle=1$ have been used. For random cascading models, $\rho_{\boldsymbol{\pi}_{j}}$ is independent of the projecting direction, therefore the index $j$ will be omitted from now on. The normalized projected density is given by

$$
\frac{\rho_{\pi}}{\left\langle\rho_{\pi}\right\rangle}=\sum_{m_{j}=1}^{n} \prod_{\alpha \in(m)} \frac{W_{\alpha}}{\lambda}
$$

Denote the cut density $\rho_{c}$ by:

$$
\rho_{C}=\prod_{\alpha \in(m)} \frac{W_{\alpha}}{\lambda}
$$


This is the density of fluctuations generated by a 1-dimensional random cascade, hence fluctuations along the projecting direction. Formula (12) yields the normalized projected density

$$
\frac{\rho_{\pi}}{\left\langle\rho_{\pi}\right\rangle}=\sum_{m_{j}=1}^{n} \rho_{C}
$$

This can be interpreted as follows. The density $\rho_{C}$ is the density of a 1-dimensional cut of the system, i.e. when densities are taken into account only in one of the 1-dimensional subtrees of the $D$-dimensional Cayley tree. Note that comparing Eq.(11) for $D$ and $D=1$, fluctuations are much bigger for the cut system, they are charaterized by the intermittency indices $\varphi_{C}$

$$
\varphi_{C}=D \varphi_{D}=\frac{\ln \left\langle W^{q}\right\rangle}{\ln \lambda}
$$

Moments of the normalized projected densities are given from Eq.(14) by

$$
\frac{\left\langle\rho_{\pi}^{q}\right\rangle}{\left\langle\rho_{\pi}\right\rangle^{q}}=\left\langle\left(\sum_{m_{j}=1}^{n} \rho_{C}\right)^{q}\right\rangle
$$

Following the thermodynamic formalism of Ref.[5], one introduces the partition function of the cut system,

$$
Z_{C}(i)=\sum \rho_{C}^{i}
$$

moments of the normalized projected densities are given by moments of the cut partition function evaluated at $i=1$

$$
\frac{\left\langle\rho_{\pi}^{q}\right\rangle}{\left\langle\rho_{\pi}\right\rangle^{q}}=\left\langle Z_{C}^{q}(1)\right\rangle
$$

Moments $\left\langle Z_{C}^{q}(i)\right\rangle$ probe the thermodynamic structure of the 1-dimensional cut system. They have been computed in the limit of large number of bins in Ref.[7] allowing a thorough investigation of the projected moments.

\section{III - PROJECTED RENYI CODIMENSIONS}

In order to compute the intermittency indices of the projected system, one thus has to compute moments of the cut partition function. Moreover the index $i$ is fixed to be 1 , the phase diagram is cut into two pieces along the line $i=1$ (see Fig.3). For small enough moments bulk intermittency (phase I) is present whereas above a certain $q_{1}$ moments probe phase II which is related to "anomalous events ${ }^{\text {[4] }}$. In each of these phases, one has the scaling law

$$
\left\langle Z_{C}^{q}(1)\right\rangle \underset{n \longrightarrow+\infty}{\propto} n^{E_{C}(q, 1)},
$$

where $n$ is the number of bins.

The exponents $E_{C}(q, 1)$ are parametrized by the analytic continuation of the free energy function $\tilde{\varepsilon}_{C}$ of the cut fluctuation patterns, namely:

$$
\tilde{\varepsilon}_{C}=\frac{1+\varphi_{C}}{q}-1
$$


They are given by

$$
\begin{array}{llll}
E_{C}(q, 1)=q \tilde{\varepsilon}_{C}(1) & \text { if } & q<q_{1} \\
E_{C}(q, 1)=q \tilde{\varepsilon}_{C}(q) & \text { if } & q>q_{1}
\end{array}
$$

where $q_{1}$ is solution of the equation

$$
\tilde{\varepsilon}_{C}\left(q_{1}\right)=\tilde{\varepsilon}_{C}(1)
$$

Notice that $\tilde{\varepsilon}_{C}(1)=0$ therefore $E_{C}(q, 1)$ is zero below $q_{1}$. This has a very natural interpretation in terms of Renyi dimensions which will be given later.

From Eq.(19) and Eq.(21) the projected intermittency indices are obtained, they are defined by

$$
\frac{\left\langle\rho_{\pi}^{q}\right\rangle}{\left\langle\rho_{\pi}\right\rangle^{q}} \underset{n \longrightarrow+\infty}{\propto} n^{(D-1)_{\varphi_{\pi}}}
$$

notice the factor $n^{D-1}$ which takes into account the $(D-1)$ dimensional volume element. The intermittency indices read

$$
\begin{aligned}
& \varphi_{\pi}=0 \quad \text { if } \quad q<q_{1} \\
& \varphi_{\pi}=\frac{1}{D-1}\left(D \varphi_{D}-q+1\right) \quad \text { if } \quad q \geq q_{1}
\end{aligned}
$$

As expected ${ }^{[8]}$ bulk intermittency which corresponds to phase I of the cut phase diagram, is wiped out by projection, i.e. the intermittency indices are zero below $q_{1}$. Notice that these are asymptotic intermittency indices, it represents the asymptotic slope of factorial moments variations as the bin size goes to zero. Therefore a zero intermittency index means that the factorial moments flattens as the bin size becomes very small. This confirms a previous analysis by Ochs ${ }^{[10]}$.

Nevertheless for high enough moments, the effect of $D$-dimensional intermittency is not eliminated. The intermittency indices (Eq.24) illustrate an intuitive geometric picture. Let us define the Renyi codimensions ${ }^{[10]}$

$$
D-\mathcal{D}=\overline{\mathcal{D}}=\frac{D \varphi_{D}}{q-1}
$$

where $\mathcal{D}$ is the Renyi dimension of the fluctuation pattern.

This is the dimension of the set of bins where intermittency takes place, as a specific dynamical structure. Similarly the Renyi codimension of the projected intermittency is given by

$$
\overline{\mathcal{D}}_{\pi}=\frac{(D-1) \varphi_{\pi}}{q-1}
$$

Then using relation $(24), \overline{\mathcal{D}}_{\pi}$ is easily computed:

$$
\begin{aligned}
& \overline{\mathcal{D}}_{\pi}=0 \quad \text { if } \quad q<q_{1} \\
& \overline{\mathcal{D}}_{\pi}=\overline{\mathcal{D}}-1 \geq 0 \quad \text { if } \quad q \geq q_{1}
\end{aligned}
$$

One can write it more compactly

$$
\overline{\mathcal{D}}_{\pi}=\operatorname{Max}(0, \overline{\mathcal{D}}-1)
$$


This is nothing but the usual fact that codimensions are additive, they decrease by exactly the number of projected dimension (here 1 ). Therefore $q_{1}$ spots out the transition from bulk intermittency where intermittency takes place on $\overline{\mathcal{D}}<1$ sets to a stronger intermittency taking place on a $\overline{\mathcal{D}}>1$ set. Fluctuations taking place on intermittent sets whose Renyi dimension is too small are irrelevant by projection.

\section{IV - PROJECTED MULTIFRACTAL SPECTRA AND THEIR CLASSIFICATION}

Local information given by intermittency indices is not enough to analyze the structure of projected events. A further tool is needed: multifractal analysis. To do so, introduce the partition function of the projected system

$$
Z_{\pi}(q)=\sum \rho_{\pi}^{q}
$$

where the sum is extended over the $n^{D-1}$ projected bins. Notice the difference with $Z_{C}(i)$ in Eq.(17). This is a random quantity which scales as $n$ tends to infinity as

$$
Z_{\pi}(q) \underset{n \longrightarrow+\infty}{\propto} n^{-\tau(q)}
$$

The exponent $\tau(q)$ is the Procaccia function ${ }^{[12]}$. It can be proven ${ }^{[6]}$ that $\tau(q)$ is related to the free energy $\varepsilon_{\pi}$.

$$
\varepsilon_{\pi}=\frac{\tau(q)}{q}=\operatorname{Min}_{q^{\prime} \leq q}\left(\frac{\varphi_{\pi}+1}{q^{\prime}}-1\right)
$$

The free energy $\varepsilon_{\pi}$ is frozen at its minimum value when the function $\frac{\varphi_{r}+1}{q}-1$ increases. This critical value is reached for a moment rank equal to $q_{C}$. Typically there is always such a freezing of the free energy $\varepsilon_{\pi}$. Notice also that following Eq.(24) $\varepsilon_{\pi}$ is defined by different expressions if $q \leq q_{1}$ and $q>q_{1}$. When the free energy freezes, it is the signal that a structural transition for the projected system takes place. It is thus the signal of a structural change of the events observed by projection.

From the free energy, one can get the multifractal spectrum $f_{\pi}\left(\alpha_{\pi}\right)$ of the projected system. This entails that as $n$ goes to infinity ( $n$ is the number of bins along one direction) the projected density $\rho_{\pi}$ scales as

$$
\rho_{\pi} \underset{n \longrightarrow+\infty}{\propto} n^{-\alpha_{\pi}}
$$

The number of dominating bins for a given value of $\alpha_{\pi}$ scales as

$$
\mathcal{N}_{\alpha_{z}} \underset{n \longrightarrow+\infty}{\propto} n^{f_{r}\left(\alpha_{*}\right)}
$$

The exponent $f_{\pi}\left(\alpha_{\pi}\right)$ is the fractal dimension of the intermittent set whose Lipschitz-Holder exponents is $\alpha_{\pi}$. The multifractal spectrum is given by ${ }^{[6]}$

$$
\begin{aligned}
& \alpha_{\pi}=(D-1)\left(1-\frac{\mathrm{d} \varphi_{\pi}}{\mathrm{d} q}\right) \\
& f_{\pi}\left(\alpha_{\pi}\right)=-(D-1) q^{2} \frac{\mathrm{d} \varepsilon_{\pi}}{\mathrm{d} q}
\end{aligned}
$$


Utilizing the known expressions for the projected intermittency indices $\varphi_{\pi}$ given by Eq.(24), one can compute the multifractal spectrum $f_{\pi}\left(\alpha_{\pi}\right)$ as a function of the original $D$-dimensional spectrum $f(\alpha)$. This reads

$$
\begin{array}{ll}
\varphi_{\pi}=(D-1) ; \quad f_{\pi}\left(\alpha_{\pi}\right)=(D-1) \quad \text { if } \quad q<q_{1} \\
\varphi_{\pi}=\alpha ; \quad f_{\pi}\left(\alpha_{\pi}\right)=f(\alpha) \quad \text { if } \quad q \geq q_{1}
\end{array}
$$

Notice that the projection does not operate on the multifractal spectrum for $q \geq q_{1}$. Therefore the geometric construction of the projected multifractal spectrum is straightforward. One has just to draw a point $(D-1, D-1)$ for $q<q_{1}$ and remove the corresponding part $q<q_{1}$ of the original $D$-dimensional multifractal spectrum $f(\alpha)$.

One can then iterate the above approach to several projections. If one performs $j$ projections, the multifractal spectrum is simply:

$$
\begin{aligned}
& \alpha_{\pi^{j}}=(D-j) \quad f_{\pi^{j}}\left(\alpha_{\pi^{j}}\right)=(D-j) \quad \text { if } \quad q<q_{j} \\
& \alpha_{\pi^{j}}=\alpha \quad f_{\pi^{j}}\left(\alpha_{\pi^{j}}\right)=f(\alpha) \quad \text { if } \quad q \geq q_{j}
\end{aligned}
$$

where $q_{j}$ is solution of the equation

$$
\overline{\mathcal{D}}=j
$$

The intermittency indices are given by the codimension law

$$
\overline{\mathcal{D}}_{\pi^{j}}=\operatorname{Max}(0, \overline{\mathcal{D}}-j)
$$

when the free energy gets frozen, the multifractal spectrum remains equal to zero.

Three cases of projection are possible depending on the relative values of $q_{c}$ and $q_{1}$.

a) Bulk intermittency $\left(\mathbf{q}_{1}=+\infty\right)$

Bulk intermittency is depicted by phase $I$ in the cut phase diagram of Fig.3. In that case, the multifractal spectrum is merely reduced to a point corresponding to the dimension of the support $(D-1)$ (see Fig.4a). One has

$$
\begin{aligned}
f_{\pi}\left(\alpha_{\pi}\right) & =D-1 \\
\alpha_{\pi} & =D-1
\end{aligned}
$$

This means that intermittency is damped by projection as found in Ref.[8]. Let us emphasize that this may even happen if the $D$-dimensional cascade admits a "non-thermal" transition $\left(q_{c} \neq+\infty\right)$. For instance, $\alpha$-models whose random weight is discrete

$$
r(W)=p \delta(W-a)+(1-p) \delta(W-b)
$$

fall within this class of damped intermittency as soon as $b<\lambda$.

b) Elementary excitations $\left(\mathbf{q}_{\mathbf{i}}>\mathbf{q}_{\mathbf{c}}\right)$

The spectrum $f(\alpha)$ is zero at $q_{1}$ (see Fig.4b). This means that projection is only sensitive to elementary excitations of the $D$-dimensional cascade. Indeed the condition $q_{1}>q_{c}$ implies that elementary excitations belong to the ground state phase of the $D$-dimensional cascade $\left(q>q_{c}\right)$. The fact that $q_{1}$ is greated than $q_{c}$ reveals that these spikes emerge by projection. These spikes are 
the projection of the $D$-dimensional patterns associated with a structural transition. Thus in the framework of particle physics, these events are probably to be attributed to the formation of quark or gluon jet structures, the transition describing the hadronization mechanism.

c) Anomalous events $\left(q_{c}>q_{1}\right)$

A continuous multifractal spectrum can only be obtained if $q_{1}$ is finite. However, another condition has to be met in order to preserve a piece of the genuine $D$-dimensional multifractal spectrum (see Fig.4c). One has to impose $q_{c}>q_{1}$. Geometrically this entails that the multifractal spectrum of the ( $D-1)$-dimensional cascade is continuous from $q_{1}$ to $q_{c}$ (it may happen that the $D$-dimensional cascade has no structural transition, i.e. there is no $q_{c}$ ).

When $q<q_{1}$ the intermittency indices are zero (flattening of factorial moments), the particle distribution $\left\{k_{m}\right\}$ is of the same type as in case a): bulk intermittency. At $q=q_{1}$ there is a first order transition (discontinuity of the multifractal spectrum). This is the transition from the regime of bulk intermittency to fluctuations localized on bigger sets, i.e. such that $\overline{\mathcal{D}}_{q_{1}}=1$. These sets $(\overline{\mathcal{D}}=1)$ are responsible for the projected intermittency. Nevertheless they do not spot out the presence of a $D$-dimensional structural transition (existence of $q_{c}$ ). The physical significance of anomalous events will be emphasized in Section V.

\section{V - APPLICATIONS}

In order to apply the above results to experimental data, we will use a convenient parametrization of intermittency indices: Levy law intermittency indices ${ }^{[11]}$.

$$
\varphi_{D}=\frac{\varphi_{2}}{2^{\mu}-2}\left(q^{\mu}-q\right)
$$

where the second intermittency exponent is $\varphi_{2}$ and the Levy index $\mu \in[0,2]$. If $\mu=2$ it is the $\log$ normal approximation whereas $\mu=0$ gives $\beta$-models ( $\alpha$-models where $a=0$ see Eq.(40)). When $\mu$ is less than one the particle dynamical fluctuations have essentially the geometry of a monofractal (a single fractal dimension governs the intermittency exponents). This has been argued to be a signal of the formation of a quark-gluon plasma in the cascade ${ }^{[13]}$. Above $\mu=1$, sheer multifractal spectra appear which seem to be compatible with cascading mechanisms as for $\mathrm{e}^{+}-\mathrm{e}^{-}$and $\mu-p$ reactions.

Let us discuss projection effects. From Eq.(41) it is easy to impose the condition $\overline{\mathcal{D}}=1$ which entails projected intermittency. Two cases are obtained. They correspond either to weak intermittency $\mu<1$ or strong intermittency $\mu>1$.

a) Weak intermittency $\mu<1$

Suppose that $\varphi_{2}$ is given and one studies the effect of varying the Levy index $\mu$. There are two distinct domains separated by the curve (see Figs.5 and 6)

$$
\varphi_{2}=\frac{2-2^{\mu}}{D}
$$

Below this curve the distribution of particles is bulk intermittent (case a), namely the projected intermittency indices are always zero. Conversely above the threshold the multifractal spectrum is continuous (anomalous events case c) but as for typical values of $\varphi_{2}$ the transition index $q_{C}$ is very large, it is practically not different from a monofractal cascade dominated by $(D-1)$-dimensional spikes. 


\section{b) Strong intermittency}

When $\mu>1$ the two cases $q_{C}>q_{1}$ and $q_{C}<q_{1}$ appear (see Section IV). The transition rank $q_{C}$ is given by ${ }^{[11]}$

$$
q_{C}=r^{-\frac{1}{4}}
$$

where $r=\frac{(\mu-1) \varphi_{2}}{2^{\mu}-2}$. The rank $q_{1}$, corresponding to $(D-1)$ dimensional spikes is obtained imposing the condition $\overline{\mathcal{D}}=1$; it is solution of the equation

$$
q_{1}^{\mu}=q_{1}+\frac{\left(2^{\mu}-2\right)}{D \varphi_{2}}\left(q_{1}-1\right)
$$

Comparing Eqs.(43) and (44), one obtains the limiting value $q_{C}=q_{1}$ when $r=r_{0}$

$$
r_{0}^{-\frac{1}{\mu}}=\frac{\theta+1}{\theta+r_{0}}
$$

with $\theta=\frac{\mu-1}{D}$. If $r<r_{0}$ then $q_{C}>q_{1}$, the multifractal spectrum is continuous, the projected particle distribution is dominated by $(D-1)$-dimensional spikes ("anomalous events"). If $r \geq r_{0}$ then $q_{C}<q_{1}$, this is the case of "elementary excitations" revealing a $D$-dimensional structural transition. We have solved numerically Eq.(45) for $D=2$ and $D=3$. This gives a diagram $\varphi_{2}$ as a function of $\mu$ (see Figs.5 and 6). Introduce the critical value $\tilde{\varphi}_{2}$ function of $r_{0}(\mu)$, solution of Eq.(45)

$$
\tilde{\varphi}_{2}=\frac{2^{\mu}-2}{\mu-1} r_{0}(\mu)
$$

The critical value $\tilde{\varphi}_{2}$ of $\varphi_{2}$ delimits two regions, if $\varphi_{2}>\tilde{\varphi}_{2}$ projection reveals a $D$-dimensional structural transition. It is striking that the intermittency threshold $\tilde{\varphi}_{2}$ is smaller in 3-dimensions than in 2-dimensions. Physically this yields that projection reveals the presence of a $D$-dimensional structural transition as soon as the second intermittency index $\varphi_{2}$ is greater than 0.2 .

\section{IV - CONCLUSION}

Analysis of projected intermittency allows one to obtain several answers to problems mentioned in the introduction. In particular, it provides an explanation to the appearance of "anomalous events", the role of bulk intermittency, and sheds light on the existence of a $D$-dimensional "non thermal" phase transitions.

\section{i) 2-dimensional intermittency}

First of all it has been recently noticed that a quasi universal value of the 2-dimensional Levy index is $\mu=1,6$ for $\mathrm{e}^{+}-\mathrm{e}^{-}, \mu-p$ reactions and Herwig Monte Carlo simulations. Taking into account for $\mathrm{e}^{+}-\mathrm{e}^{-}$a second intermittency index $\varphi_{2} \sim 0,17$ gives $r \sim 0,1$ which is smaller than $r_{0}(1,6)$. Therefore "anomalous events" are expected for these reactions if intermittency is two dimensional. However one expects that, at least for $\mathrm{e}^{+}-\mathrm{e}^{-}$reactions, elementary excitations such as quark-gluon jets should pop out. A hint that intermittency may be 3-dimensional. Let us analyze 3-dimensional intermittency. 


\section{ii) 3-dimensional intermittency}

We can interpret the observed NA22 "anomalous event". This is a 2-dimensional spike which is rather delocalized in azimuth and overall transverse momentum $p_{T}$. Therefore it can be seen as the signal of a three-dimensional intermittency characterized by $\varphi_{2} \leqslant 0,2$. Notice that $\varphi_{2} \sim 0,2$ (around the threshold) is what has been numerically obtained recently ${ }^{[14]}$ for hadron-hadron collisions. It is also expected that for $\mathrm{e}^{+}-\mathrm{e}^{-}$channels one has ${ }^{[14]} \varphi_{2} \gtrsim 0,6$. This agrees with our physical intuition that elementary excitations such as quark-gluon jets should be responsible for these intermittent patterns.

Eventually, the case $\mu<1$ is relevant to heavy ion reactions. For instance the observation of "anomalous events" in heavy ion reactions would make unlikely the creation of a quark-gluon plasma in these reactions. Indeed, projected intermittency of a quark-gluon plasma requires that particle distributions are bulk intermittent (second order phase transitions are characterized by a monofractal structure). This means that flattening of projected moments at small rapidity bins should take place for all moments indices.

Theoretically, it would be extremely valuable to obtain intermittency indices directly from field theory and thus predicts the dimensional structure of multiparticle events. This goal is beyond reach at present even though intermittency analysis is a step in this direction.

One of us (Ph. Brax) wants to thank John Ellis and the Theory Division at CERN for its hospitality during part of this year. 


\section{REFERENCES}

[1] A. Bialas and R. Peschanski, Nucl. Phys. B 273 (1986) 703.

[2] NA22 collaboration, Ajinenko et al., Phys. Lett. B 222 (1989) 306.

[3] JACEE collaboration, T.H. Burnett et al., Phys. Rev. Lett. 50 (1983) 2062.

[4] A. Bialas, A. Szczerba and K. Zalewski, Z. Phys. C 46 (1990) 163.

A. Bialas and K. Zalewski, Phys. Lett. B 238 (1990) 413.

[5] R. Peschanski, Nucl. Phys. B 327 (1989) 144.

[6] P. Brax and R. Peschanski, Nucl. Phys. B 346 (1990) 65-83.

[7] P. Brax and R. Peschanski, Saclay preprint SPhT/90-044, to appear in Nucl. Phys. B.

[8] W. Ochs, "Intermittency and cascade processes" presented at the Santa Fe Workshop (1990) on intermittency in high energy collisions.

W. Ochs, "Multidimensional intermittency analysis", MPI PAE/PTh 63/90.

[9] S. Gupta et al., "The search for intermittency in the finite size Ising Model" BeTP90/21. Ivo Novak, "Intermittent behaviour in the Ising model", Bratslava preprint 1989.

A. Bialas and J. Seixas, CERN preprint TH-S757/90.

[11] P. Brax and R. Peschanski, Physics Lett. B 253 (1991) 225.

[12] T.C. Halsey et al., Phys. Rev. A 33 (1986) 1141.

[13] A. Bialas and R. Hwa, "Intermittency parameters as possible signal for quark gluon plasma formation", CERN preprint TH-S754/90.

[14] J. Seixas, private communication, and A. Bialas, J. Seixas, Ref.[8]. 


\section{FIGURE CAPTIONS}

Figure 1: The shape of two drastically different multiparticle production events representing the density of particles as a function of rapidity.

a) NA22 event coined as an "anomalous event". It is delocalized in azimuth and transverse momentum.

b) JACEE event where many dips and spikes appear. A typical case of bulk intermittency.

Figure 2: The Cayley tree on which independent realizations of the random variable $W$ are assigned. The branching ratio is $\lambda^{D}$ (here equal to $2^{2}$ ) and the different end points are denoted by $[m]=\left(m_{1}, \ldots, m_{D}\right)$ where $m_{j} \in\{1, \ldots, n\}$. Path $[m]$ is shown by a thicker line, a branch $\alpha$ being depicted.

Figure 3: The phase diagram of the 1-dimensional cut cascade. Within boxes are written the exponent $E_{C}(q, i)$ such that $\left\langle Z_{C}^{q}(i)\right\rangle \propto n^{E_{C}(q, i)}$. The relevant line is $i=1$ which sweeps two regions I and II. In region I $\tilde{\varepsilon}_{C}(1)=0, q_{1}$ is the signal of the appearance of "anomalous events" in the cut cascade responsible for the intermittency in the projected system (region II).

Figure 4: The three possible projected multifractal spectra.

a) Flat distributions (bulk intermittency).

b) For $q_{1}>q_{C}$ the bistable case which reveals a $D$-dimensional structural transition (elementary excitations).

c) The multifractal spectrum of a $(D-1)$-dimensional model for which $q_{C}>q_{1}$ (anomalous events).

Figure 5: The three different types of intermittency in 2-dimensions as a function of $\varphi_{2}$ and the Levy index $\mu$.

a) Bulk intermittency.

b) Elementary excitations.

c) Anomalous events.

Figure 6: Ditto as in Fig.5 for intermittency in 3 dimensions. Notice that the transition line between elementary excitations b) and anomalous events c) is almost constant as a function of $\mu$. 

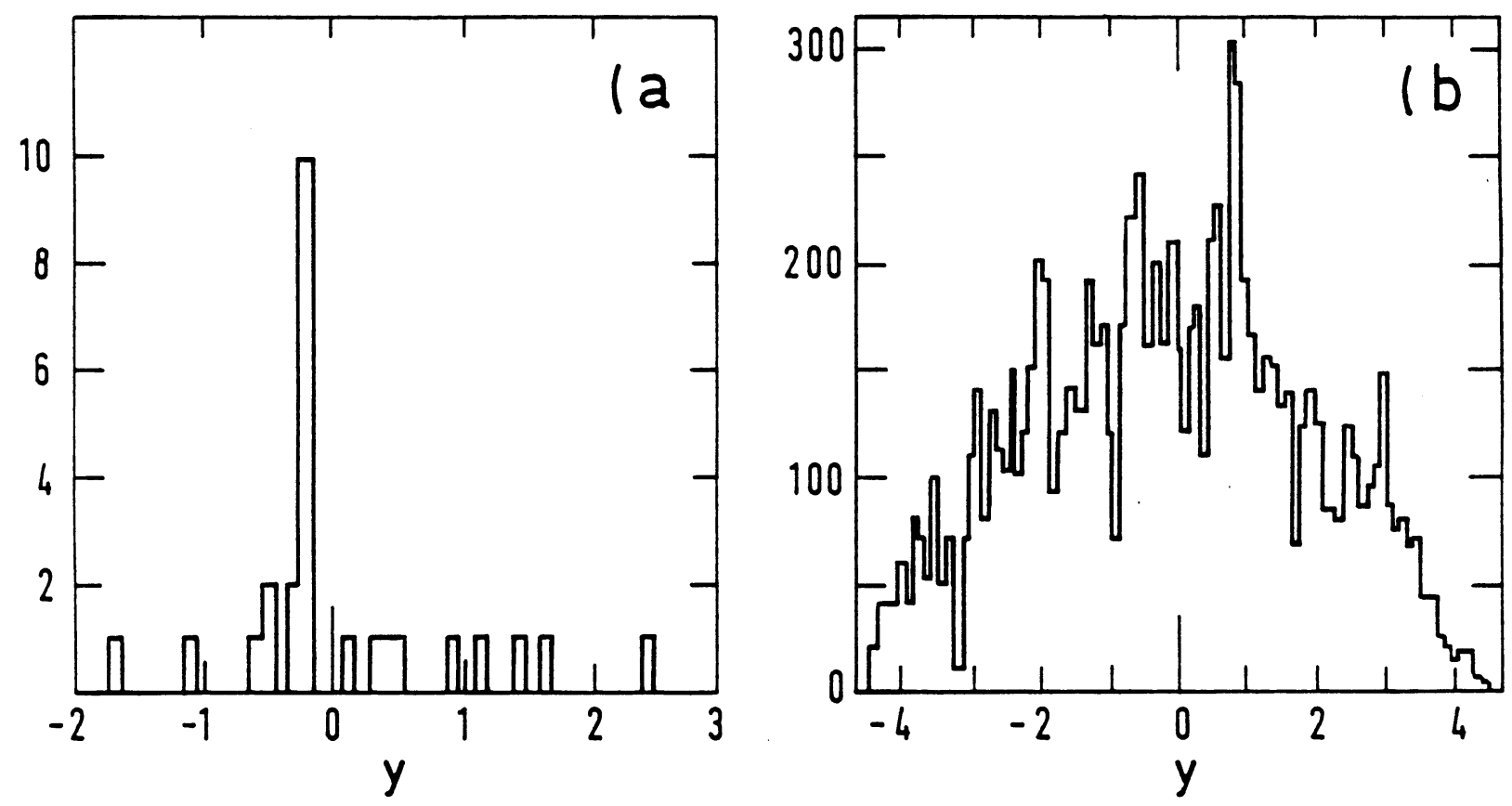

Fig. 1 


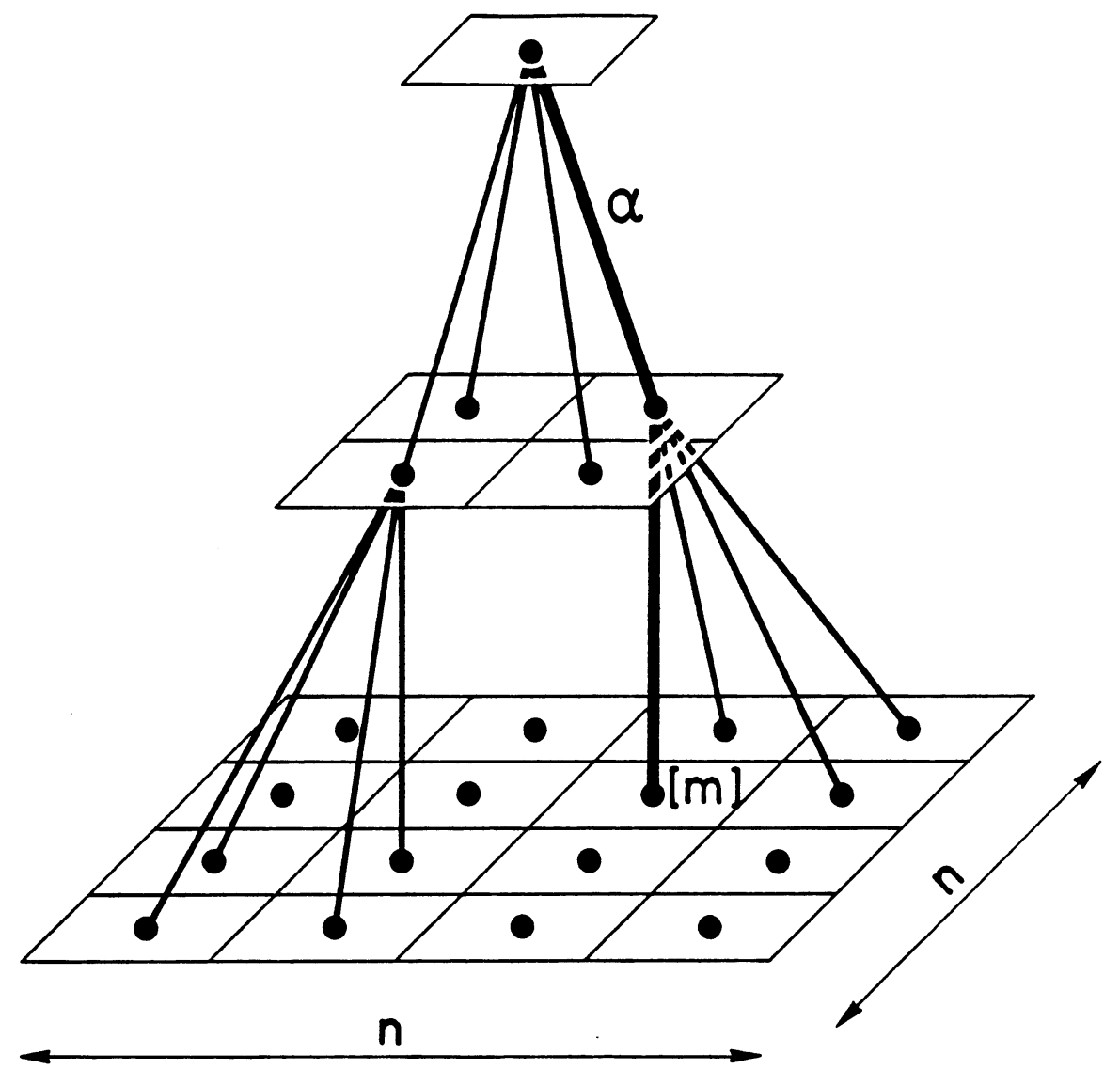

Fig. 2 


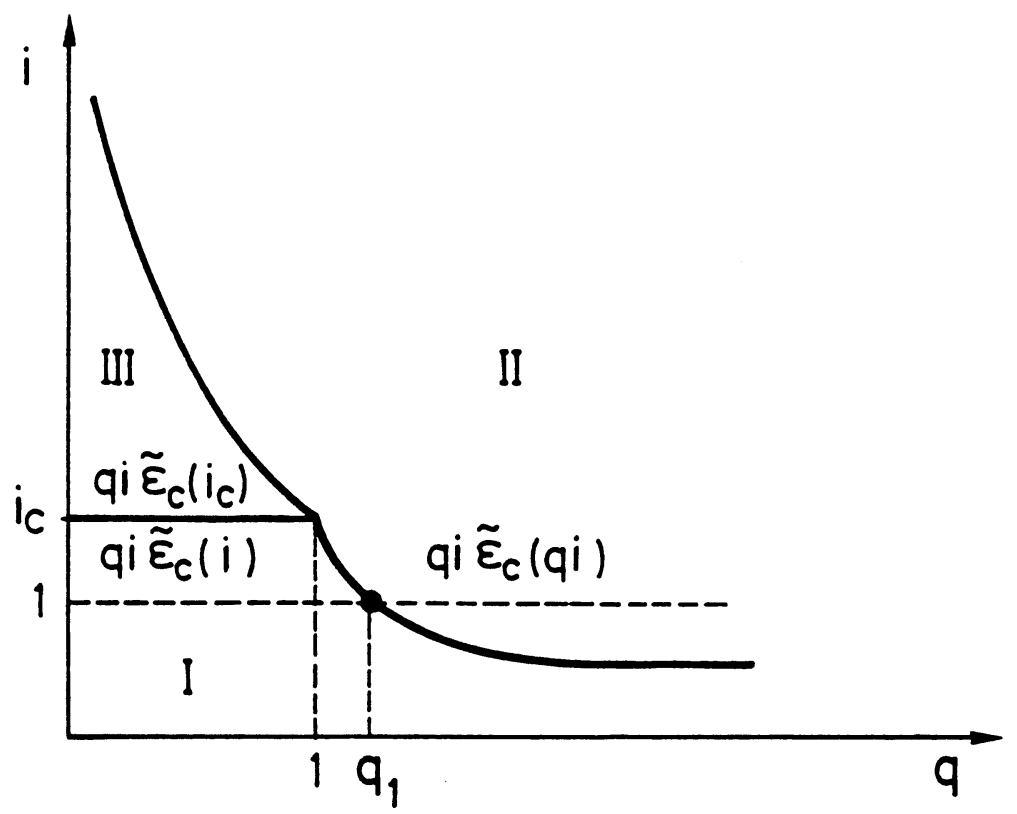

Fig. 3 

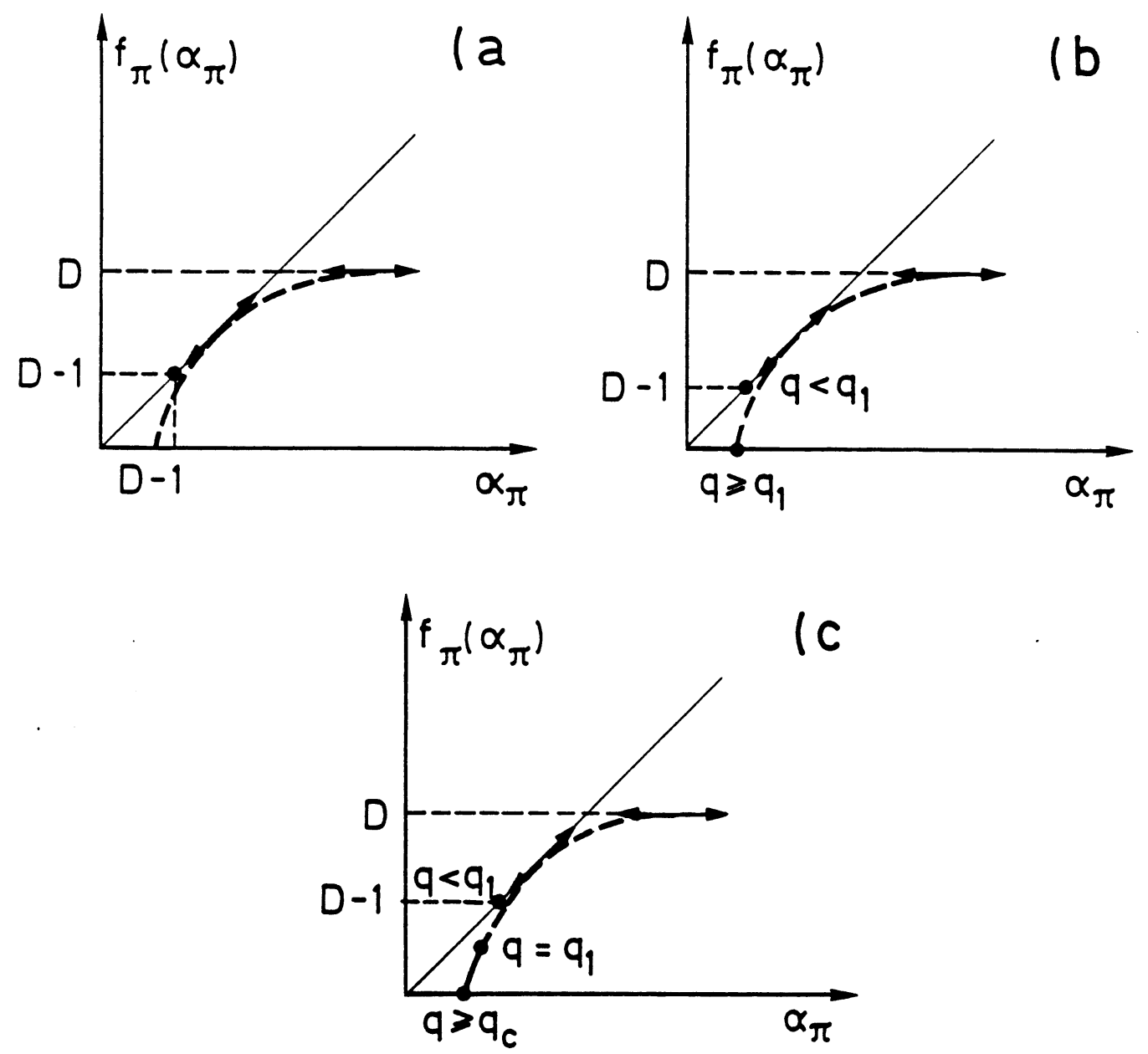

Fig. 4 


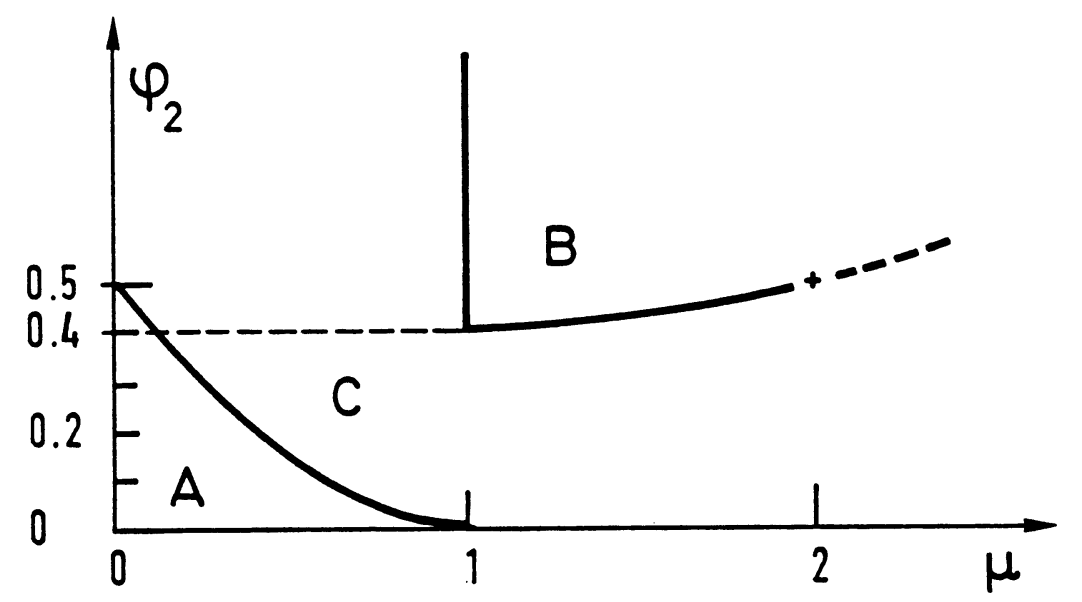

Fig. 5 


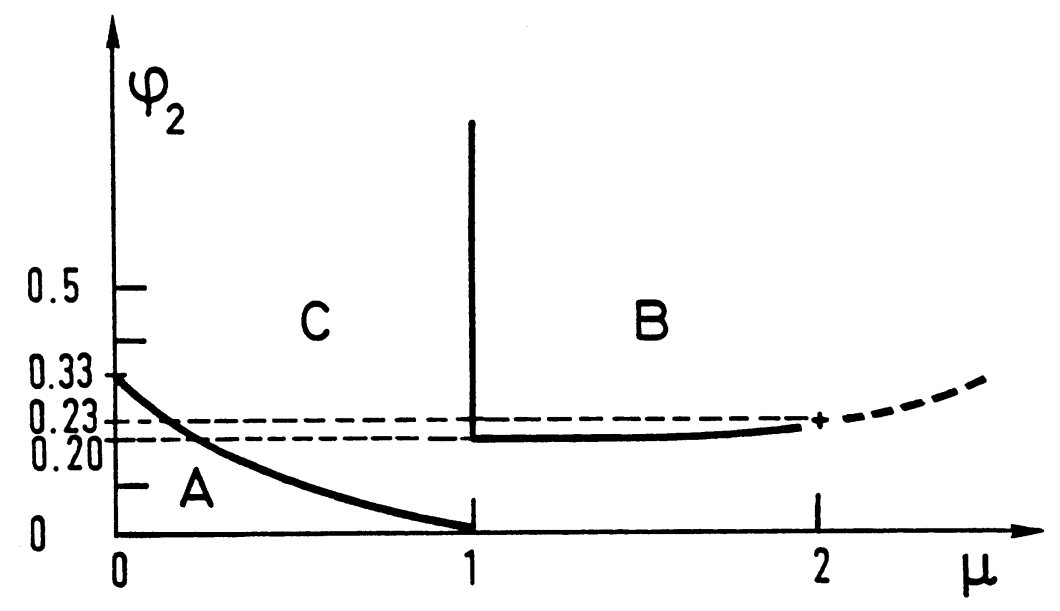

Fig. 6 